\title{
What is the role of imaging in acute low back pain?
}

\author{
Humaira Lateef · Deepak Patel
}

Published online: 28 April 2009

(c) The Author(s) 2009. This article is published with open access at Springerlink.com

\begin{abstract}
In patients with non specific acute low back pain, without the red flags, a conservative approach is preferable, with assessment in 4-6 weeks. The natural history of low back pain is favorable with improvement over time, thus reassurance to such patients is very important. However, a plain radiograph or more advanced imaging techniques like MRI/CT may be ordered in back pain associated with radiculopathy or spinal stenosis and back pain associated with progressive neurologic deficits. There is limited role of imaging in non specific acute low back pain without the red flags, as the findings correlate poorly with symptoms.
\end{abstract}

Keywords Back pain - Herniated disk - Spondylolysis · Spondylolisthesis - Vertebral compression fracture .

Bone scan · Lumbar MRI

\section{Introduction}

Acute low back pain is one of the most common conditions encountered in primary care. It affects two-thirds of adults at sometime in their lives [1]. The lifetime prevalence of low back pain has been reported to be $70-85 \%$ [2]. In majority of the cases, acute back pain is self limited and benign with no cause identified in $95 \%$ of the patients. In such patients the cause is either a muscular or ligamentous

H. Lateef · D. Patel ( $₫)$

Flower Hospital Family Medicine Residency, Sylvania,

OH, USA

e-mail: Deepak_S_Patel@rsh.net

D. Patel

The Toledo Hospital Primary Care Sports Medicine Fellowhsip,

Toledo, OH, USA injury $[3,4]$. When acute back pain is associated with neurologic symptoms, then an extensive work up is warranted to look for causes like herniated intervertebral disk, spinal stenosis, and cauda equine syndrome, which accounts for only $5 \%$ of acute back pain cases [1]. A focused history and physical examinations is the first step to determine the specific underlying conditions and to look for evidence of neurologic involvement [5, 6]. This helps in categorizing patients in three broad categories: non specific low back pain, back pain associated with radiculopathy, or spinal stenosis and back pain associated with another specific spinal cause. The latter category includes patients with serious neurologic deficits or underlying condition requiring immediate evaluation such as tumor, infection, or cauda equine syndrome as well as patients with other conditions that may respond to specific treatment such as vertebral compression fractures or ankylosing spodylitis [7].

Herniated disks, which are often managed initially like lumbar strains, account for only $4 \%$ of acute back pain cases. Out of this $4 \%$ about $95 \%$ of patients with herniation have sciatica; therefore, the likelihood of a symptomatic herniated disk in a patient with acute back pain but no symptoms of sciatica is approximately one in 500 [4].

Although, the incidence of vertebral compression fractures increases with advancing age, it accounts for less than $5 \%$ of acute back pain $[4,8]$. The vertebral compression fracture may be the cause of sudden onset of acute back pain in patients older than 50 years of age. The history may not be helpful in diagnosing vertebral compression fracture, as the fractures may occur without any increased force on the spine.

There is insufficient evidence for intervals or methods for reassessment of history and physical exam. However, a 4 week follow-up is generally recommended as most 
patients with acute back pain improve in the first month after initial presentation [7]. Although imaging is commonly used for further evaluation, it should not be considered as a replacement of clinical suspicion based on an accurate history and physical exam. It is important to keep in mind the limitations of the diagnostic studies and to consider how the management will be influenced by the information obtained from these studies.

\section{Diagnostic imaging}

In primary care, the most commonly used imaging modalities are X-ray, MRI, CT, and nuclear medicine bone scan. Sometimes, more advanced imaging techniques are used in anticipation for surgery and that includes CT myelography and PET scans [9].

\section{Plain radiographs}

The most commonly ordered spinal imaging test is X-ray because of ready availability and low cost. As most cases of mechanical low back pain resolve with conservative treatment, an X-ray is rarely indicated as part of initial workup. There is no evidence to prove that obtaining $\mathrm{X}$-rays is associated with better patient outcomes [10, 11]. $\mathrm{X}$-rays are helpful for evaluation of fracture, bony deformity including degenerative changes, sacroiliitis, disk and vertebral body height, and assessment of bony density and architecture. X-rays are part of initial workup, if the history and physical exam suggests non mechanical cause of back pain or if red flags are present (Table 1) [9, 12].

In most cases, anteroposterior (AP) and lateral views are the first line of approach, as higher radiation exposure is associated with oblique films. Plain AP and lateral radiographs are also the initial imaging study obtained for a suspected compression fracture [8]. The compression

\section{Table 1}

Red flags suggesting further investigation

Fever,

Age $>50$,

Recent trauma,

Pain at night or at rest,

Progressive motor or sensory deficit,

Saddle anesthesia,

Unexplained weight loss,

History of cancer or strong suspicion of cancer,

History of osteoporosis or chronic steroid use,

Immunosuppression or

Failure to improve after 6 weeks of conservative therapy. fractures tend to occur at multiple levels, so it is important to radiograph the entire spine. Oblique films are ordered when there is suspicion of spondylolysis, as suggestive from history and physical examination. Oblique views show the pars interarticularis in profile and thus helps in the diagnosis of spondylolysis [1]. In these images the defect in the pars may become evident by looking for the fracture of the neck of the "Scottie dog" (Figs. 1, 2). In patients with possible spondylolisthesis or prior spinal surgery, flexion, or extension films should be obtained (Fig. 3) [13].

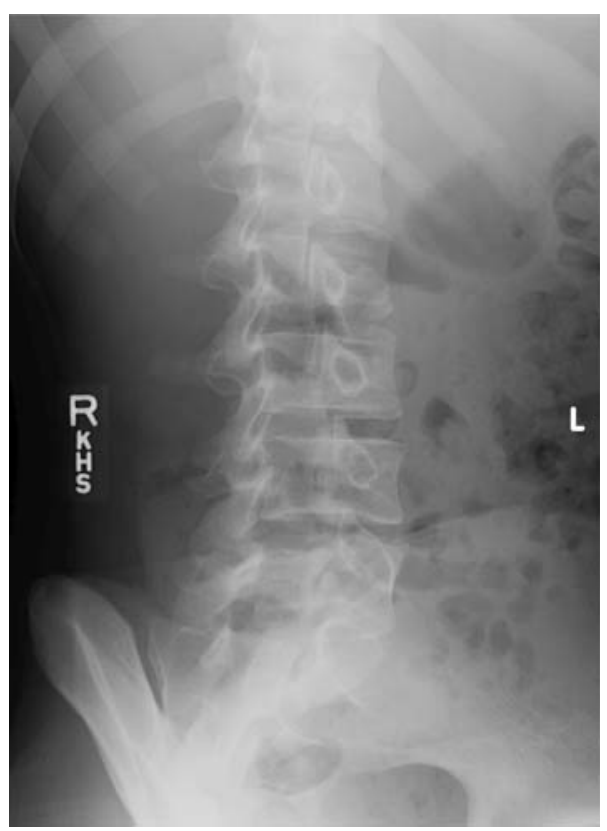

Fig. 1 Left oblique image of L5 Spondylolysis

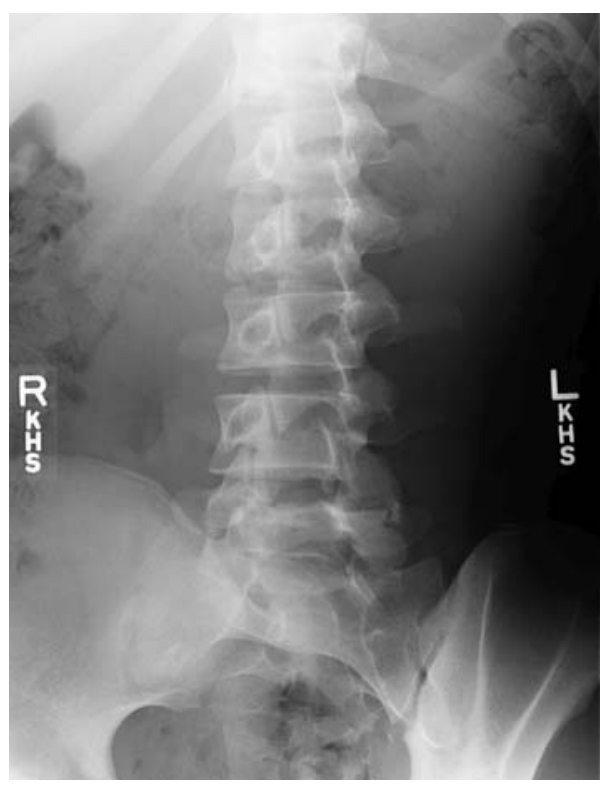

Fig. 2 Right oblique image of L5 Spondylolysis 


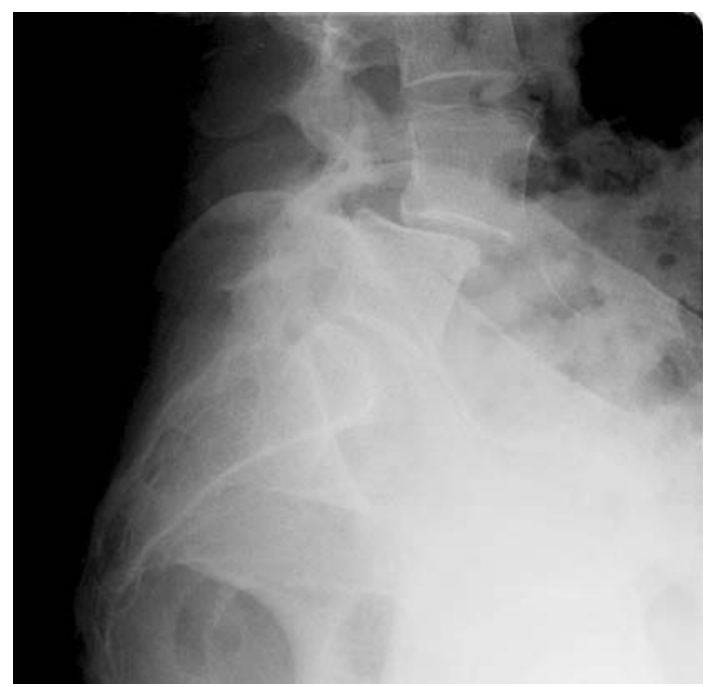

Fig. 3 Spondylisthesis of L4 on L5

A drawback of lumbar radiography is exposure of gonads to ionizing radiation, especially with oblique view or multiple exposures. Another drawback of lower back $\mathrm{X}$-ray in acute back pain are the identification of certain abnormalities, like facet joint abnormality or mild scoliosis, that are only incidental findings and are unrelated to the back symptoms as most of these conditions are seen in persons without back pain [14]. Plain radiographs can not visualize disks, are not sensitive for herniated disk, and are not helpful in diagnosing nerve root impingement [1]. Despite these limitations, radiographs are commonly recommended prior to proceeding with more advanced imaging (MRI/CT) [1].

\section{Magnetic resonance imaging and computed tomography}

MRI or CT is recommended in patients with severe or progressive neurologic deficits or with serious underlying conditions, such as vertebral infection, cauda equine syndrome, or cancer with spinal cord compression [7]. In these conditions, delayed diagnosis is associated with poorer outcomes.

In patients presenting with acute back pain with signs or symptoms of herniated disk or a systemic disease, MRI or CT is rarely indicated except in patients with a strong suspicion of cancer or infection or cauda equine syndrome based on their history and physical examination $[12,15]$. Most patients with herniated disk improve with conservative therapy. As shown by a few small studies, healing and regression occurs in herniated portion of the disk with time, as seen on serial MRI [15]. A small number of patients with significant pain or neurologic deficit even after 6 weeks of

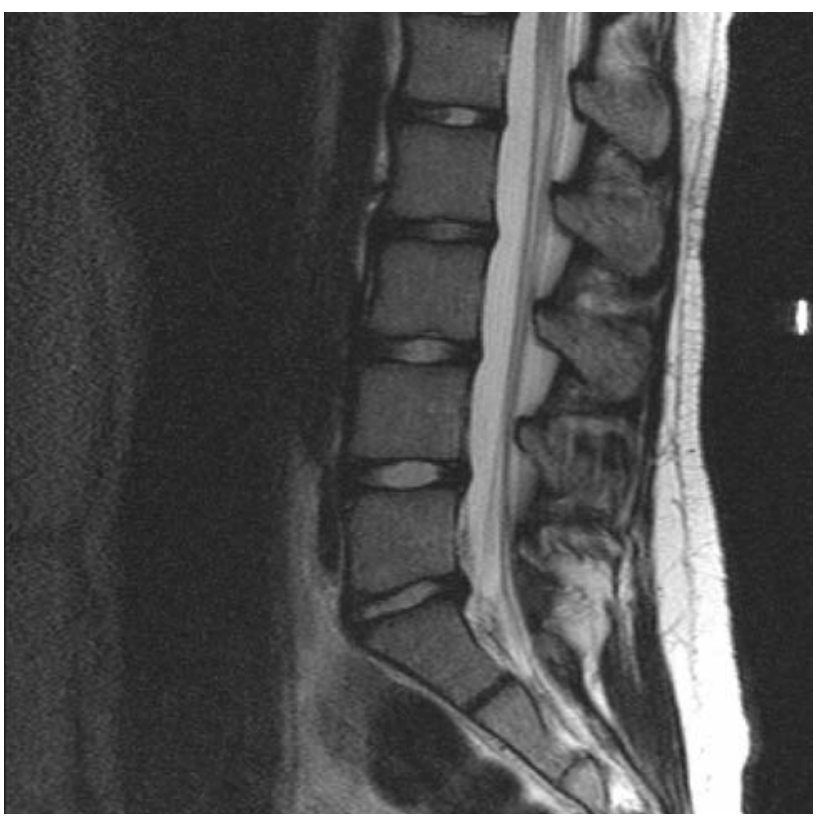

Fig. 4 MRI of disk bulge at L5-S1

conservative therapy will require an MRI or CT in consideration of surgery [15].

MRI does not require radiation exposure and provides better visualization of soft tissue and spinal canal, and thus preferred over CT [1].

Routine advanced imaging is also not associated with improved patient outcomes. Many radiographic abnormalities are detected with MRI and CT that are poorly related with the symptoms and sometimes such incidental findings leads to unnecessary additional workup or intervention. MRI studies identified $22-40 \%$ of adults with herniated disk, who are asymptomatic and pain free, but one study found $81 \%$ of such asymptomatic individuals with a bulging disk (Fig. 4) [1]. Thus, a MRI should not be ordered to evaluate for the presence of a herniated disk as an initial step in the diagnosis as it is more commonly an incidental finding and may not be the cause of pain. An initial conservative approach is more cost-effective and medically appropriate in acute low back pain for the first 46 weeks. The MRI becomes more valuable for evaluating patients with persistent radicular symptoms despite conservative treatment.

Computed tomography (CT) has superior depiction of cortical bone than MRI. This is a disadvantage of the MRI that it cannot directly visualize cortical bone. Thus, when bony anatomy is critical, CT is preferable. In patients with acute trauma, CT may be better in visualizing fractures, especially of the posterior elements [1]. CT is also more reliable than MRI for detecting facet degenerative changes, because of the same reason [16]. Both CT and MRI are useful in evaluating vertebral compression fracture. CT is 
Fig. 5 Bone scans of L3 Spondylolysis

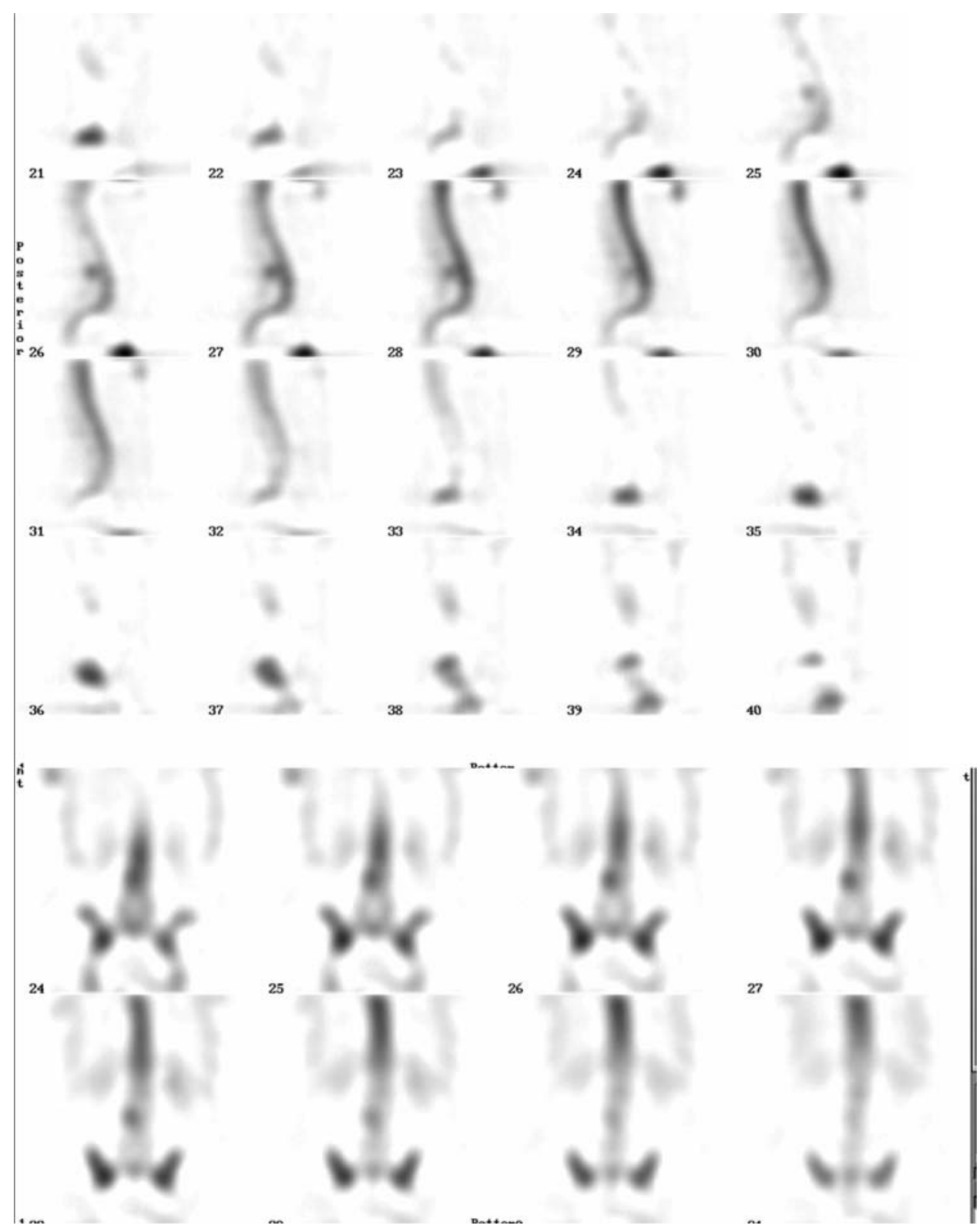

the next choice after a negative radiograph in vertebral compression fracture, but an MRI is preferred, if neurologic symptoms are present [8].

\section{Bone scan}

Bone scans are used mainly to detect occult fractures, stress fractures, infections, or bony metastases and to differentiate them from degenerative changes [1]. Bone scans also can differentiate between an acute versus healed compression fracture because new fractures will appear "hot" [8]. It has limited role in diagnosing acute low back pain but is the test of choice to detect stress fracture of the pars interarticularis or symptomatic spondylolysis (Fig. 5). Technetium bone scans are very sensitive for stress fractures, but are non specific, a normal bone scan virtually excludes the diagnosis [17]. A bone scan may remain positive for up to 6-9 months after the inciting event in Spondylolysis [18].

\section{Conclusions}

In patients with non specific acute low back pain, without the red flags, a conservative approach is preferable, with assessment in 4-6 weeks. The natural history of low back pain is favorable with improvement over time, thus 
reassurance to such patients is very important. However, a plain radiograph or more advanced imaging techniques like MRI/CT may be ordered in back pain associated with radiculopathy or spinal stenosis and back pain associated with progressive neurologic deficits. There is limited role of imaging in non specific acute low back pain without the red flags, as the findings correlate poorly with symptoms.

Open Access This article is distributed under the terms of the Creative Commons Attribution Noncommercial License which permits any noncommercial use, distribution, and reproduction in any medium, provided the original author(s) and source are credited.

\section{References}

1. Jarvik J, Deyo R. Diagnostic evaluation of low back pain with emphasis on imaging. Ann Intern Med. 2002;137:586-95.

2. Andersson G. Epidemiological features of chronic low back pain. Lancet. 1999;354:581-5.

3. Atlas SJ, Deyo RA. Evaluating and managing acute low back pain in the primary care setting. J Gen Intern Med. 2001;16:12031.

4. Kinkade S. Evaluation and treatment of acute low back pain. Am Fam Physician. 2007;75(8):1181-8. (Apr 15).

5. Deyo RA, Rainville J, Kent DL. What can the history and physical exam tell us about low back pain? JAMA. 1992;268: 760-5.

6. Bigos S, Bowyer O, Braen G, Brown K, Deyo R, Haldeman S, et al. Acute low back pain problems in adults. Clinical Practice Guidline ns 14. AHCPR Publication No. 95-0642. Rockville, MD. US Department of Health and Human Services; 1994. Available at http://www.chirobase.org/07Strategy/AHCPR/ahcprclinician. html.
7. Chou R, Qaseem A, Snow V, Casey D, Cross T, Shekelle P, et al. Diagnosis and treatment of low back pain: a joint clinical practice guideline from the American College of Physicians and the American Pain Society. Ann Intern Med. 2007;147(7):478-91.

8. Old JL, Calvert M. Vertebral compression fractures in the elderly. Am Fam Physician. 2004;69(1):111-6. Jan 1.

9. Harwood MI, Smith BJ. Low back pain: a primary care approach. Clin Fam Pract. 2005;7(2):279-303.

10. Deyo RA, Diehl AK, Rosenthal M. Reducing roentgenography use. Can patient expectations be altered? Arch Intern Med. 1987;147:141-5.

11. Kerry S, Hilton S, Dundas D, Rink E, Oakeshott P. Radiography for low back pain: a randomized controlled trial and observational study in primary care. Br J Gen Pract. 2002;52:469-74.

12. Chiodo A. Acute low back pain. In: Low back pain guideline update. University of Michigan Health System; 2003. Available at http://cme.med.umich.edu/pdf/guideline/backpain03.pdf. Accessed Mar 2008.

13. Lehrich JR, Katz JN, Sheon RP. Approach to the diagnosis and evaluation of low back pain in adults; 2006.

14. VanTulder MW, Assendelft WJ, Koes BW, Bouter LM. Spinal radiographic findings and non-specific low back pain. A systemic review of observational studies. Spine. 1997;22:427-34.

15. Deyo RA, Weinstein JN. Low back pain. N Engl J Med. 2001;344:363-70.

16. Schwarzer AC, Wang SC, O'Driscoll D, Harrington T, Bogduk $\mathrm{N}$, Laurent R. The ability of computed tomography to identify a painful zygapophysial joint in patients with chronic back pain. Spine. 1995;20:907-12.

17. Johnson AW, Weiss CB Jr, Stento K, Wheeler DL. Stress fractures of the sacrum. An atypical cause of low back pain in the female athlete. Am J Sports Med. 2001;29:498-508.

18. Micheli LG, Curtis C. Stress fracture in the spine and sacrum. Clin Sports Med. 2006;25:75-88. 